\title{
Oriole Beta
}

\section{A Parametric Solution for Robotic Motion Design Using Animation}

\author{
Ebrahim Poustinchi ${ }^{1}$ \\ ${ }^{1}$ Kent State University \\ Impoustin@kent.edu
}

This paper presents a project-based research study using the beta version of Oriole-a custom-made animation-based plug-in for grasshopper $3 D$ visual programming environment, to develop robotic motion/controlling solutions. Oriole, as a parametric tool, makes it possible for designers/users to "design"-instead of generating, the motions of the robot based on the notion of keyframing and time-based animation. Through the use of Oriole, users can simulate-and ultimately develop robotic motions/performances in more intuitive ways. This unique feature enables users with minor or no programming background to create robotic solutions using Oriole as a software/plugin Bridge. Using Rhinoceros $3 D$ as a digital modeling platform in conjunction with Grasshopper $3 D$ and its robotic simulation platforms, Oriole can develop controlling strategies for different industrial robots such as $K U K A, A B B$, and Universal Robots. Oriole enables designers to create a precise interaction between the robot, its spatial "performance" and the physical environment, through animation and keyframing to "design" robotic interactions and movements as frames of animation instead of segments of a curve "path."

Keywords: Robotics, Software Development, Animation, Parametric Design, Design

\section{INTRODUCTION \\ Robotics in Design}

In the past few years and after the introduction of robotics to design disciplines, many designers and architects started to use robots to physicalize the outcome of their digital design process; the use of CNC (Computer Numerical Control) machines in the design discipline is an example. Later, given the five to eight-axis freedom of robots, multiple designers, architects, and academic set-ups started to rethink the potential of this new tool and possibly "medium" in identifying novel making/production processes. Projects such as "Robotic Softness" (Brugnaro, G., et al., 2016), Bartlett RC4 and multiple "chair" projects (Soler, V., Retsin, G., Jimenez, M. G., 2017), and ICD/ITKE Research Pavilions-2013-14 research pavilion for instance (Yunis, L., et al., 2014), to name a few, are challenging the conventional ways of thinking about robots in the realm of fabrication through new means of communication or making. In 
addition, projects such as "Mobile Robotic Fabrication System for Filament Structures" (Yablonina, M, et al., 2017), and similar projects, also question the limitations of robots/robotics themselves, as a customizable tool to do a "customized" fabrication task.

Although novel robotic fabrication and making methods are always welcome in the field of digital design/fabrication and are crucial for the creative design world, there is a missed opportunity to use the performative aspects of robots as an active agent where the robotic motion itself can be "designed." In another word, in some of the mentioned projects and similar ones, although the process is tremendously innovative, the performance of the robot follows conventional thinking methods of "engineered" efficiency in finding the optimized "path" to perform a task or avoid any accidental collisions. These optimizations are the result of an engineered robotic controlling/programming platforms and mindsets that are native to industry.

Later as a response the mentioned controlling needs, some professions, academic set-ups, and creative platforms started to develop new methods for interacting with robots through design software platforms with a focus on designing the motion of the robot as well as its functional operation. Esperant.O platform at Sci-Arc by Kruysman-Proto (Testa, $P, 2017)$, BDMove at Bot and Dolly and UCLA, by Bot and Dolly, and RobotAnimator (Ibrahim, K., 2017) are some examples of these intuitive controlling platforms. The "Impossible Objects" design series by Kruysman-Proto and Curime Batliner (Kruysman, B., Proto, J., 2014) are some examples of designing the motion/performance of the robot while addressing the operation of the required task.

Similarly and as part of the special-effect production of the movie Gravity, Bot and Dolly-creative robotic studio used the idea of the curated motion of the robot to animate the camera, light, images, and performers (Bot \& Dolly, 2018).

Although these robotic motion design platforms and projects had a priceless impact on "creative" robotics, most of them are restricted to highly cus- tomized software platforms that are limited to the developer's design studio. In another word, despite the universal impact of these tools on the contemporary discourse of creative robotics, none of these "animation-based" robotic controlling platforms were/are open to the public. Oriole research and Oriole Beta, as a parametric robotic controlling platform, seeks the potential of similar methodologies in controlling robots, in more conventional/open source yet creative design platforms such as Rhino and Grasshopper 3D. Using "familiar" design software platforms, and in the context of an advanced graduate research studio, Oriole Beta investigates the potentials of animation-based robotic motion control as a possible medium to study and design dynamic architectural spaces.

\section{Animation in Design}

In the past three decades and after the introduction of digital design, architecture and other design disciplines started to adopt the changes and shiftstriggered by the digital wave, as design opportunities. Following this digital movement, and as a cultural shift in design, in his book Animate From, Greg Lynn calls for attention to the possibilities of animation as a design-tool in architecture (Lynn, G., 1999). Immediately after, animation became an accepted important experimental architectural design, simulation, and representation tool through which, animation concepts-such as deformation, blend-shapes, constraining, keyframing, graph-editing and gradual transformations, and physic simulations-to name a few, grown into architectural, formal and spatial strategies and languages. As a result of this growth and in today's conversation about design and architecture, animation and its frame of mind, play a serious role when it comes to design thinking.

\section{Oriole Beta}

When developers create design software platforms for designers/architects, most of the developers look at the culture of design as a platform for communication and experimentation-if possible. Introduction of node-based visual programming platforms such as 
Autodesk MAYA's node system, Grasshopper 3D, and Touch-Designer for instance or developing hardware controllers such as a 3D mouse, Palette controlling interface and NKNM platforms (Poustinchi, E., Wang, S, Luhan, G., 2018), are a few examples where developers expand the possibilities of a classic programming language or conventional analogue or digital input platforms through intuitive/visualized interactive software/hardware architecture to amplify the experimental and intuitive aspects of the design medium. This goal is usually achieved through redefining/rethinking the interaction scenarios between the user and the software/hardware platform.

Following this notion of "targeted" software development-for designers, and in the light of close similarities between animation design and robotic motion-controlling process (discussed in further detail below), Oriole Beta seeks a solution to make it a possible, for designers to "animate" robots as means of controlling and interacting with robot as a potentially a creative medium. By rapidly growing desire and culture of robots as a team member in innovative design and fabrication set-ups, it seems to be essential to have controlling methods through design software platforms, to use animation strategies to design robotic motions.

By investigating design students' and designers' experiences in using Oriole Beta platform, this research paper illustrates the possible design opportunities made available due to the use of a keyframebased parametric animation controlling platform for robots. The proposed method enhances the design experience with robots not only as fabrication/production tools but also as a new outlook to interactively design and evaluate the physical performance of the robot as potentially a solution for a tangible/physical animated design process (architectural design UI) as well as architecture and space as an outcome of the design process (architectural UX).

Building on previous experiences in the field of animation-in design disciplines, as well as robotic controlling solutions, Oriole Beta proposes new controlling solutions for robots within a familiar 3D de- sign software (Rhinoceros) and with a familiar technique/process (animation keyframing).

\section{RESEARCH QUESTIONS AND METHODS}

Developed based upon earlier studies in animationbased robotic controlling solutions, Oriole Beta-as a part of ongoing research, questions two potentials for design-oriented animation-based robot controlling:

1. How an animation-based parametric robotic controlling platform in design software, can reorient/expand the use of a robot, from being a fabrication tool to possibly becoming a design medium?

2. How moving from code-based/path-based robotic programming to animation-based motion design-as a more intuitive way of designing robotic motion, diversifies the users of the robots-as design tools/mediums and beyond, especially with it comes to users with a minor technical background?

To study these questions with a border range of audience and potential users, Oriole Beta has been developed as an independent open-source plug-in, separate from the robotic/controlling software/plug-ins and act as an addition to digital robotic simulation components. In another word, independent from the robotic software/plug-in that generates the movecommands, Oriole Beta can serve as a bridge, which animates and converts key-framed positions into understandable data for the robotic post-processor (discussed in further detail below). This freedom/flexibility makes it possible for the end-user to use Oriole to customize the robotic motion for any robots-KUKA, $A B B$ and Universal to name a few, as long as there is a post-processor available for them in Grasshopper 3D environment.

Developed to be used in the context of a research studio at the College of Architecture and Environmental Design, at Kent State University, Oriole Beta has four main components: (a) Inverse Kinematic (IK) Direct Frame, (b) Key Frame Solver, (c) Wait Solver, 
and (d) Robot Lab (Fig. 1). However, to use Oriole Beta and to complete a physical project, there is a need for a robot arm and a grasshopper-based robotic post-processor plug-in (Fig. 2). However, as one of the distinctive features of Oriole Beta and since it has been developed as a collaborative plugin, Oriole Beta can be used in conjunction with any other parametric robot controlling platforms (postprocessors) in Grasshopper 3D such as KUKA|prc, $\mathrm{HAL}$, and Taco-to name a few.

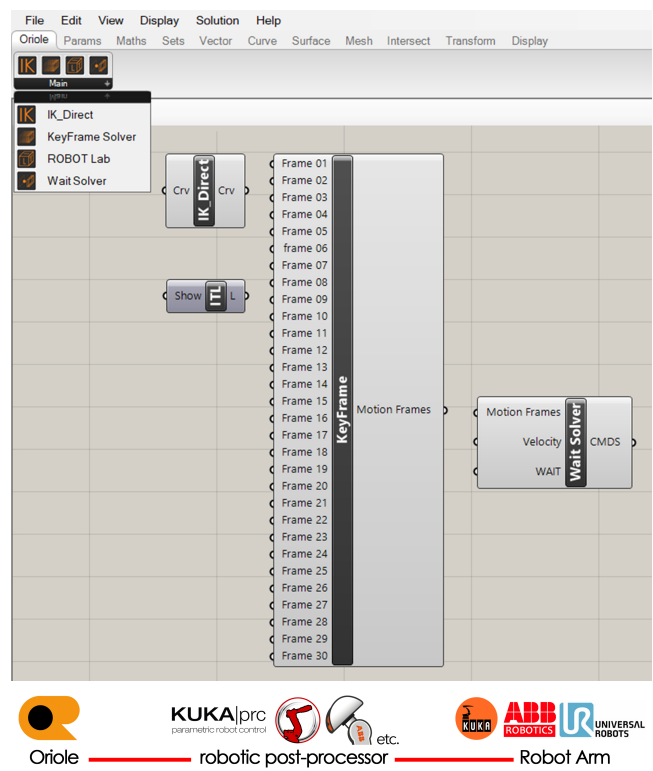

For the current research, we produced a platform to use Oriole Beta as a part of a graduate design research studio, using KUKA KR6 R900 Sixx robot arm and with a focus on the robotic motion as a medium to study the potential of three-dimensional architectural reconfigurability. Initiated at the desktop scale, students explored the possibility of a robotically animated architectural massing compositionwith a focus on a fundamental question of changeable relationships between inside/outside. Besides, some students used Oriole as a method to study for- mal, spatial, and compositional qualities of the design through videography of the physical model and as a feedback system to inform the digital design.

\section{ORIOLE: A PARAMETRIC SOLUTION FOR ANIMATION-BASED ROBOTIC MOTION DE- SIGN}

As part of the promises of Oriole research, Oriole Beta serves as a plug-in for the node-based visual programming environment of Grasshopper 3D in Rhino 3D software. Early studies with Oriole-presented in this paper as Oriole Beta, demonstrates the potential of a version of the plug-in, as a tool to animate robots in the context of a design studio, looking into the possibilities of architecture as a moveable threedimensional puzzle. Oriole Beta contains four main Oriole components, as well as KUKA|prc (Braumann, J., Bell-Cokcan, S., 2015) as the robotic post-processor plug-in and KUKA KR6 R900 Sixx as the robot arm.

\section{Oriole Beta Component: Inverse Kinematic (IK) Direct Frame}

To develop robotic motion based on animation/keyframing, one of the critical tasks is to create Key-frames, to later convert them as "position planes" into understandable axis configurations for the robot. As the first Oriole Beta component, Inverse Kinematic (IK) direct frame, uses the notion of inverse kinematics (IK)-as a familiar animation terminology to define target planes, to interactively calculate the configuration of the robot joints/axis based on the desired position of the robot "endeffector"-tool attached to the sixth axis-in six-axis robot arms. The significant advantage of this motion design method is to control the robot more spatially/three-dimensionally. Using the IK frame component, Oriole Beta users can design and save different positions and orientations for the robot end-effector in the format of an adjustable frame without moving each axis of the robot individuallyknown as forward kinematics (FK) (Fig. 3).
Figure 1

An overview of Oriole-custom plugin/components in Grasshopper 3D environment.

Figure 2

Oriole can provide 3D information for most of the available robotic post-processors in Grasshopper 3D to generate readable commands for KUKA, ABB, and Universal Robots. 
Figure 3

Difference between IK and FK solutions for robotic motion. IK interactively moves all the joints/axis of the robot to "solve" the position/orientation of the end-effector, however in FK solution; users can control the robot through the rotation of each axis.

Figure 4

Key Frame Solver calculates the transitional frames between the keyed frames as a way to define the "resolution"/smoothness of the robotic motion.

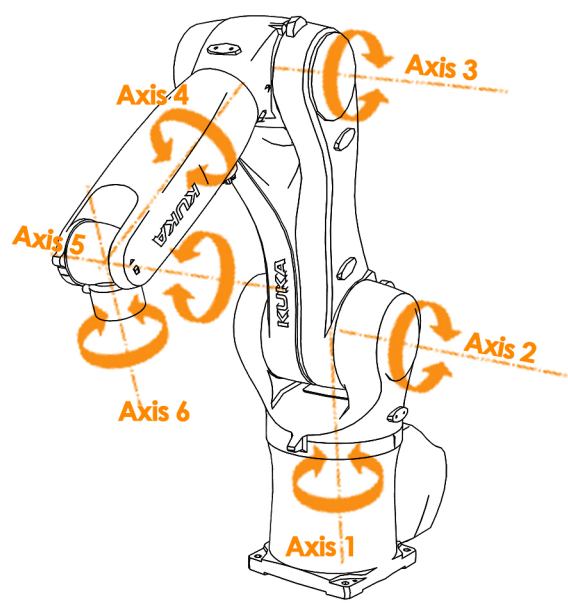

Forward Kinematic (FK) Solution

Oriole Beta Component: Key Frame Solver Using the developed IK frames by the first Oriole component, users can use the Key Frame Solver component to generate a robotic animation. Through this method, Oriole Beta users can assign 30 different IK frames to the solver, and make a robotic animation between those frames using KUKA|prc postprocessor (discussed in further detail below). Key Frame Solver uses linear animation logic to calculate the transitional frames between the "keyed" frames. Frame Solver uses linear mathematical equations to equally divide the "transformation" between frames similar to other digital animation platforms (Fig. 4).

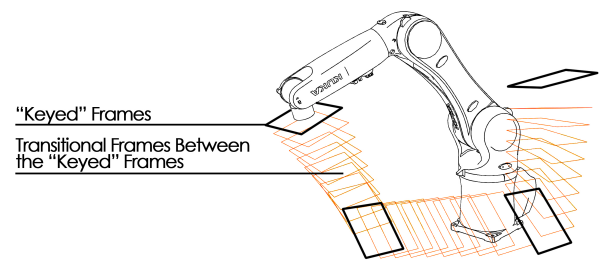

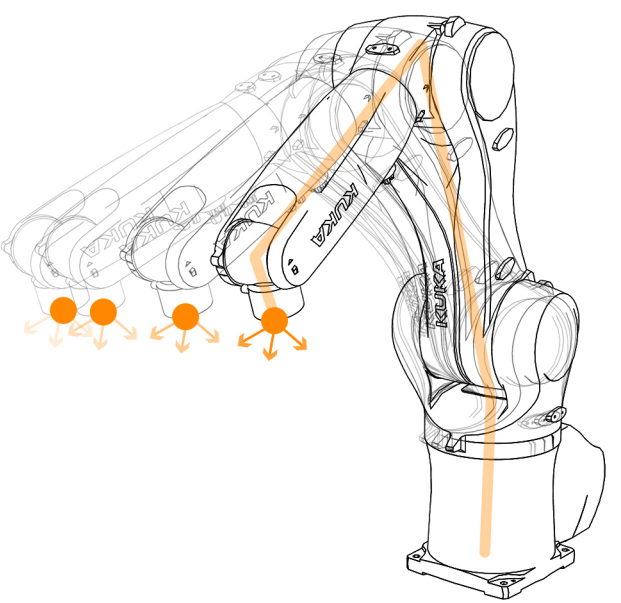

Inverse Kinematics (IK) Solution

\section{Oriole Beta Component: Wait Solver}

Given the nature of animation as a time-based medium, the third Oriole Beta component is the wait solver. It defines the wait duration-in seconds, for each of the keyed/IK frames. Using the wait solver, the robot stops for a specific period at the desired animation frame and continues with normal speed (set on the robot) during the other frames. For KUKA robots, this component embeds a KRL (KUKA robot language) wait commend into the final KRL code.

\section{Oriole Beta Component: Robot Lab}

To simulate the physical environment of the robotic experience as a way to avoid accidental collisions, Oriole Beta offers a possibility to use the digital model of the robotic set-up as a collider in grasshopper 3d environment.

\section{Robotic Post-Processor: KUKA|prc}

Although Oriole can work in conjunction with any robotic post-processor in Grasshopper 3D, for Oriole Beta we used KUKA|prc as one of the most popular KRL post-processors for KUKA robots in Grasshop- 
per. In this iteration, to make the platform more usable for non-programmer users, we integrated some of KUKA|prc components into Oriole Alpha components (IK Frame, Key Frame Solver, and Wait Solver). Later, users could use these components in conjunction with KUKA|prc native components to generate a final KRL code to run the robot.

\section{Robot Arm: KUKA KR6 R900 Sixx}

To complete an Oriole project, there is a need for a robot arm. For Oriole Beta project, we used a KR6 R900 Sixx robot arm which perfectly suits the desktop scale of our design exploration in the design research studio called "Hetero[Animo]geneous" (discussed in further detail below).

\section{OPERATION AND TEST}

To test Oriole and its architectural potential, we used Oriole Beta as a part of a graduate studio at the College of Architecture and Environmental Design, at Kent State University. Using Oriole Beta's possibility to "design" the robotic motion as potentially an architectural active agent in the process, students looked at both literal and phenomenal motion (Lynn, G. 1999) through robotics as a design medium. As part of Hetero[Animo]generous design studio, students closely looked at the possibility of robotically animation architectural space-at massing resolution, and its configurations based on different/sequential scenarios. To investigate this idea at the desktop scale, the studio looked at the canonical spatial concepts of inside/outside and through the lens of "super-component" (Wiscombe, T., 2014) as a vehicle to examine some of the initial conceptual designs. Each of these compositional studies had a super-component, which could serve as part of the primary composition or detach from the whole and live as an individual object (Fig. 5).

Throughout the process, each student studied the potential of super-component as a moving chunk of the architectural composition using the Oriole Beta's keyframing simulation solutions. Through this method, students were able to animate the super- component using IK frame component of Oriole. This feature enabled a precise animation keyframing as a way to define the exact position for the supercomponent. Using the keyframe solver component, this exact position then translated into joint/axis configurations for the robot and ultimately physical robotic motion (Fig. 6).

Through an iterative process of different simulations using Oriole Beta and developing multiple configurations based on architectural/compositional potentials of the design, students started the production of the final physical models using additive fabrication methods (3D printing and composite fabrication techniques) to initiate the physical simulation set-up. Using the Robot-Lab component of the Oriole Beta, students precisely measured the position of the physical model in relation to the actual robotbased on the digital simulation. Placing the model in its designed position/set-up, each student used their Oriole animation to activate the KUKA|prc plugin in Grasshopper 3D to generate the KRL code. As the last step of the process, students attached the super-component-moving chunk of the architectural proposal, as the end-effector to the robot with exact measurements similar to the digital configuration.

After matching the digital and physical set-ups in a back and forth process, and adjusting the differences-digitally or physically, using KUKA|prc students exported the KRL code to run the robot and animated the super-component to physically study their proposals of reconfiguring inside-outside (Fig. 7).

\section{CONCLUSION}

Although Oriole research is at its early stages of development, as a parametric animation-based grasshopper plug-in for motion design and robotic controlling, it enables designers to use robotics as a medium for design, instead of a production tool. The initial experimentation with Oriole Beta showed that even students with a minor-or no, programming background could quickly learn how to design a robotic interaction based on their familiarity 
Figure 5

As part of the

studio scenario and

in response to

Wiscombe's call for

super-component,

studio revisited part

of an architectural

composition that

can be a part of the

whole (object),-and

through some

re-configurations, a

single whole

(object).

Figure 6

The precision of

Oriole enabled

students to design

the robotic

animation/motion

digitally, with

almost no issues

when it translated

to physical robotic

motion.

Figure 7

An example of the robot moving the super-component precisely as digitally animated.
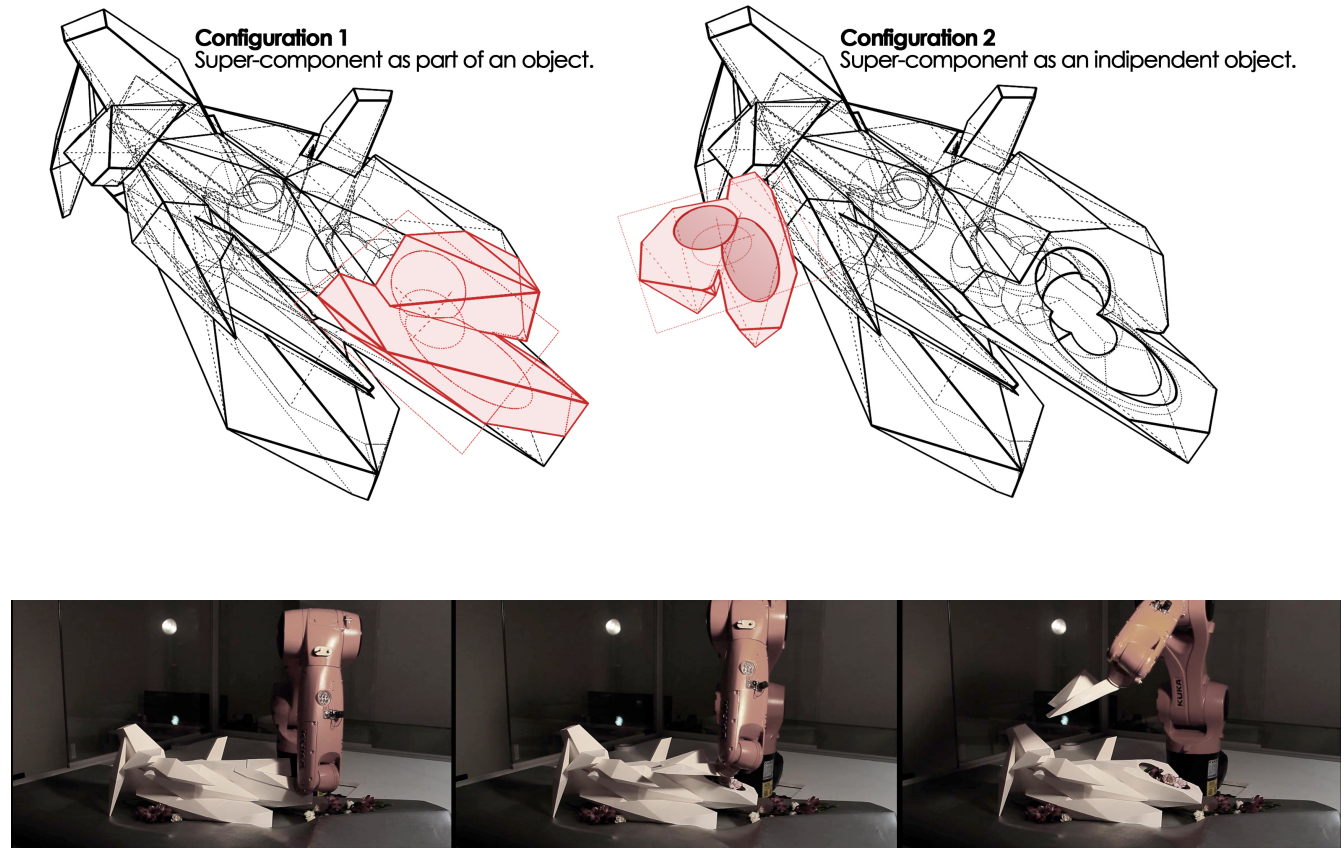

with Rhino 3D software. This flexibility/accessibility makes it possible for designers in different fields to use Oriole-and ultimately, robots, as a creative design tool.
One of the significant features of the Oriole project-Oriole Beta in this paper, is that it enables users to "design" the motion of the robot independent from the "motion path." In another

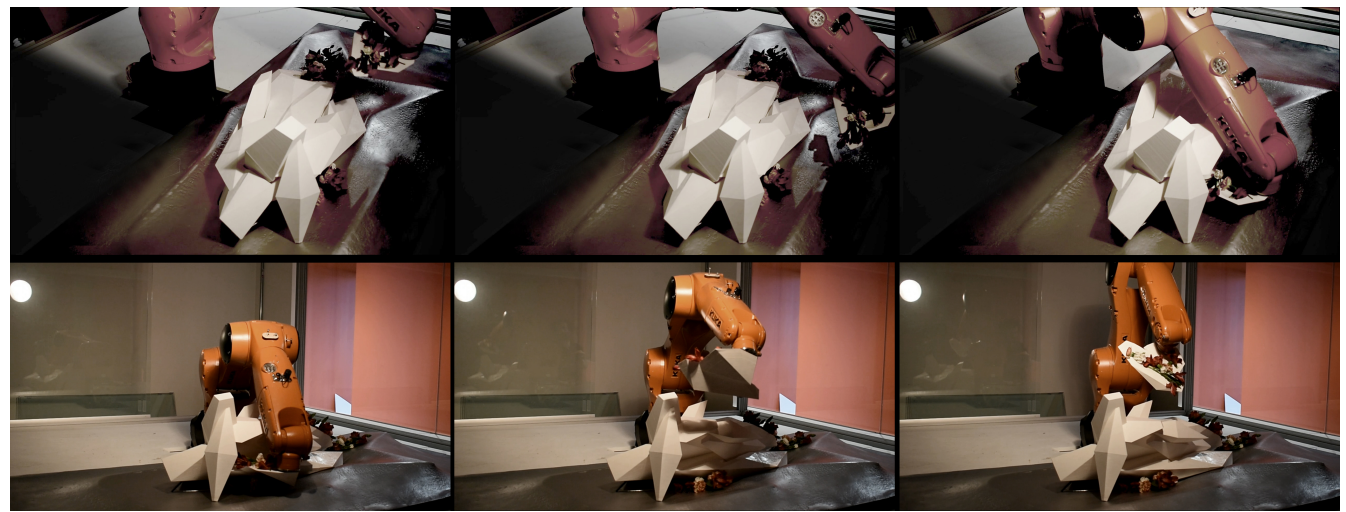


word, using Oriole, designers can "design" the position of the end-effector step-by-step. This possibility is especially critical when it comes to nonfabrication/production use of robots. In custom pick-and-place set-ups, experimental fabrications, 3D puzzle scenarios, and robotic videography to name a few, it is crucial to have control over the precise position of the end-effector and Oriole opens up possibilities to design those correct positions through keyframing and animation.

\section{LIMITATIONS AND SUGGESTIONS FOR FU- TURE RESEARCH}

Although the Oriole Beta project had invaluable impacts on developing the Oriole research, it had many limitations and missed opportunities that we are trying to address in future research investigations.

One of the main limitations-crucial to examine the precision of some of the outcomes is the number and pool of the users. For the next iterations, we will explore possibilities of an Oriole system, for a border range of users in multiple design disciplines and with different levels of experience.

Another limitation/missed opportunity for Oriole Beta that is limiting the project from fulfilling all the promises of an Oriole system is the limitation of the experience to KUKA KR6 R900 Sixx robot. For next explorations, we have started to examine the potential of Oriole in conjunction with other robots and robotic post-processors in grasshopper. Achieving this goal would tremendously increase the number of possible users.

In addition, we are currently exploring the possibilities of Oriole in real-time as an interactive bridge for robotic motion design through grasshopper 3D. Another feature that is now under development is the ability to increase control over the "transitional" frames in the keyframe solver component. Currently, the mathematical equation divides the frames inbetween frames equally. We are developing a new Oriole component to control the animation "curve" to customize the transitional speeds.

Oriole research is at its early stages of develop- ment, and we hope that next iterations have new features to expand-even more, the possibilities of robotics as a design medium for architects and designers.

\section{REFERENCES}

Braumann, J and Brell-Cokcan, S 2015 'Adaptive robot control: new parametric workflows directly from design to KUKArobots', Proceedings of the 33rd international conference on Education and Research in Computer Aided Architectural Design in Europe (eCAADe), Vienna, Austria, pp. 243-250

Brugnaro, G, Baharlou, E, Vasey, L and Menges, A 2016 'Robotic Softness: An Adaptive Robotic Fabrication Process for Woven Structures', Proceedings of the 36th Annual Conference of the Association for Computer Aided Design in Architecture (ACADIA), Ann Arbor, MI, USA, pp. 154-163

Kruysman, B and Proto, J 2014, 'Impossible objects', Architectural Design, 1(227), pp. 106-111

Lynn, G 1999, Animate FORM, Princeton Architectural Press, New York

Poustinchi, E, Wang, S and Luhan, G 2018 'NO KEYBOARD, NO MOUSE-Hybrid Digital-Analog Hardware Design for Enhancing Design UI and UX', Proceedings of the 23rd CAADRIA Conference, Beijing, China, pp. 535-544

Soler, V, Retsin, G and Jimenez, M. G. 2017 'A Generalized Approach to Non-Layered Fused Filament Fabrication.', A Generalized Approach to Non-Layered Fused Filament Fabrication. In: Proceedings of the 37th Annual Conference of the Association for Computer Aided Design in Architecture (ACADIA), Cambridge, MA, USA , pp. 562-571

Testa, P 2017, Robot House, Thames \& Hudson Inc. , New York, NY

Wiscombe, T 2014, 'Discreteness or towards a flat ontology of architecture', Project, pp. 34-43

Yablonina, $M$, Prado, $M$, Baharlou, $E$ and Schwinn, $T$ 2017, 'Mobile Robotic Fabrication System for Filament Structures', in Menges, A, Sheil, B, Glynn, R and Skavara, M (eds) 2017, Fabricate 2017, UCL Press, London, pp. 202-209

Yunis, L, Kyjánek, O, Dörstelmann, M, Prado, M, Schwinn, T and Menges, A 2014 'Bio-inspired and fabricationinformed design strategies for modular fibrous structures in architecture, Fusion.', Proceedings of the 32nd eCAADe Conference, Newcastle upon Tyne, England, UK, pp. 423-432

[1] www.andyrobot.com

[2] https://www.roboticsbusinessreview.com/ 